area was newly established in the adult area, and a 25-bed ward for emergency hospitalized patients was opened.

Results: The number of patients visiting the ED increased from 77,078 to 87,927 . The proportion of patients who returned home without treatment significantly decreased from $11.5 \%$ to $0.9 \%(\mathrm{p}<0.001)$. The number of adult patients increased from 40,814 to 60,720 , but the number of patients who could be treated on the bed decreased (22,166 (54.3\%) vs. $17,776(29.3 \%), p<0.001)$. The number of pediatric patients was similar in both periods. Median ED length of stay (LOS) of total patients increased from $193.0 \mathrm{~min}$ to $205.8 \mathrm{~min}$ ( $\mathrm{p}<0.001)$. Of the 18,900 hospitalized patients during postperiod, 1,255 (6.64\%) were admitted to the emergency ward, and the boarding (from admission decision to hospitalization) time of the admitted patients decreased from $239.2 \mathrm{~min}$ in the pre-period to $190.9 \mathrm{~min}$ in the post-period by $38.3 \mathrm{~min}$. However, more time was required for admission decision in the post-period ( 216.8 vs. $253.3, \mathrm{p}<0.001)$.

Discussion: The ED expansion allowed more patients to be treated, and the emergency ward reduced boarding times of admitted patients. However, due to the increase in the number of patients, the time required for medical treatment increased.

Prehosp Disaster Med 2019;34(Suppl. 1):s123-s124

doi:10.1017/S1049023X19002668

\section{The Effects of Current Cold Chain Management Equipment in Controlling the Temperature of Pharmaceutical Stores in an Australian Defence Force Exercise Environment}

Ms. Liz Daly

The Australian Army, Albury, Australia

Aim: The purpose of this pilot study was to analyze the current cold chain storage methods of Class 8 stores, specifically thermolabile medications and temperature sensitive diagnostics, dressings, and fluids, for the Australian Army in a training area within Australia. This research was designed to identify deficiencies in current storage methods, including the inability to maintain the recommended storage temperature of pharmaceutical stores in accordance with the Therapeutic Goods Administration, as well as foster communication between key stakeholders, including the Royal Australian Army Medical Corps and the Department of Defence Joint Health Command, and to develop a cold chain protocol specific for the Australian Defence Force.

Methods: This pilot study identified the common occurrence of breaches in a specific climate and recommends that current mission essential equipment be replaced. It also discusses the need for clearly defined guidelines with accountability of the stakeholders to ensure that the provision of health support to all Australian Defence Force personnel is in accordance with civilian standards.

Results: This pilot study identified that the carried thermolabile medications and temperature sensitive diagnostics, dressings, and fluids were commonly exposed to temperatures outside the range recommended by the manufacturers. These findings related mainly to the storage equipment for Class 8 stores used by the Army. As a result, it is recommended that such equipment is replaced so that the cold chain storage meets the Therapeutic Goods Administration Guidelines to ensure that health support to Australian Defence Force Personnel in the field is in accordance with the standard of care expected at a civilian health facility.

Discussion: This pilot study has enabled the Australian Defence Force to qualify and quantify the temperature exposure of the medications and stores and engage with key stakeholders to trial and apply new technologies and processes for the management of the cold chain.

Prehosp Disaster Med 2019;34(Suppl. 1):s124

doi:10.1017/S1049023X1900267X

\section{The Efficacy of Blood Lactate on Predicting the Prognosis of Patients with Multiple Trauma \\ Miss Di Hao \\ West China Hospital, Chengdu, China}

Introduction: Because of the rapid progress of multiple trauma patients, the early mortality rate is high. Therefore, early assessment of the severity and prognosis of multiple injuries is crucial for timely treatment and improvement of prognosis. So we need to find parameters related to mortality and severity of multiple trauma.

Aim: To find out parameters related to mortality and severity of multiple trauma.

Methods: This was a single center, trauma registry based, observational cohort study. Data were collected from consecutive patients with multiple trauma who presented to the emergency department of a tertiary referral hospital between April 2015 and December 2016. The main outcomes studied were 28-day in-hospital mortality, 24-hours mortality, emergency operation rate, and ICU admission rate.

Results: 444 patients were eventually included in the study, including 337 males (75.9\%) and 107 females (24.1\%). The 28-day survival group consisted of 381 patients (85.8\%) and the death group accounted for the other 14.2\%. Multivariate logistic regression analysis showed that heart rate, peripheral oxygen saturation, lactic acid, partial pressure of carbon dioxide, plasma albumin, hematocrit, and Glasgow score were independent risk factors for 28-day mortality. The area under the ROC curve (AUC) of the above indicators was $0.669,0.547,0.868$, $0.512,0.740,0.627$, and 0.815 , and the AUC value of lactate was the maximum.

Discussion: When the body suffers from severe trauma, it loses a lot of blood and reduces the circulating blood volume, which leads to absolutely insufficient hemoglobin content and hypoxia of tissue cells. The plasma lactate content increases at this time. Therefore, lactate can be used as an important prognostic parameter for patients with multiple trauma. In addition, we can use lactate to revise the existing trauma score to enhance its effectiveness. Prehosp Disaster Med 2019;34(Suppl. 1):s124 doi:10.1017/S1049023X19002681 